Supporting information for

\title{
Efficient Synthesis of Imidazoles From Aldehydes and 1,2-Diketones Using Microwave Irradiation
}

\author{
Scott E. Wolkenberg,* David D. Wisnoski, William H. Leister, Yi Wang, Zhijian Zhao, \\ and Craig W. Lindsley \\ Department of Medicinal Chemistry, Technology Enabled Synthesis Group, Merck \\ Research Laboratories, PO Box 4, West Point, PA 19486 USA
}

\section{General procedure for the preparation of 2,4,5-trisubstituted imidazoles (2-(4- fluorophenyl)-4,5-dipheny-limidazole):}

Benzil (42 mg, $0.2 \mathrm{mmol}$ ), 4-fluorobenzaldehyde ( $25 \mathrm{mg}, 0.2 \mathrm{mmol}$ ), and ammonium acetate $(154 \mathrm{mg}, 2.0 \mathrm{mmol})$ were combined and dissolved in $1.0 \mathrm{~mL}$ of HOAc in a $2 \mathrm{~mL}$ Smithsynthesizer ${ }^{\mathrm{TM}}$ reaction vial (Part \#352016) containing a magnetic stir bar. The reaction vessel was heated in the Smithsynthesizer ${ }^{\mathrm{TM}}$ reactor cavity for $5 \mathrm{~min}$ at $180{ }^{\circ} \mathrm{C}$, after which the vessel was rapidly cooled to $40{ }^{\circ} \mathrm{C}$ by the unit. The reaction mixture was added dropwise to a $0{ }^{\circ} \mathrm{C}$ concentrated $\mathrm{NH}_{4} \mathrm{OH}$ solution and immediately formed a white precipitate which was collected by filtration and washed with $\mathrm{H}_{2} \mathrm{O}$. The solid was dried in a vacuum oven for $18 \mathrm{~h}$ at $50{ }^{\circ} \mathrm{C}$ to afford 2,4,5-triphenylimidazole as a bright white solid (61 mg, 97\%). Analytical LCMS: single peak (2.411 min, $\mathrm{CH}_{3} \mathrm{CN} / \mathrm{H}_{2} \mathrm{O} / 0.1 \%$ TFA, 4 min gradient $)>95 \%$ pure by UV $(214 \mathrm{~nm})$ and $100 \%$ pure by ELSD. ${ }^{1} \mathrm{H}$ NMR (400, DMSO-d $)_{6} \delta 12.69(\mathrm{~s}, 1 \mathrm{H}), 8.14(\mathrm{~d}, J=2.0 \mathrm{~Hz}, 1 \mathrm{H}), 8.12(\mathrm{~d}, J=2.1 \mathrm{~Hz}, 1 \mathrm{H}), 7.55-7.21$ (m, 12H); HRMS $m / z 315.1304\left(\mathrm{C}_{21} \mathrm{H}_{15} \mathrm{FN}_{2}+\mathrm{H}^{+}\right.$requires 315.1292).

\section{Representative analytical data for entries in Table 2:}

Entry 3: Analytical LCMS: single peak (2.536 min, $\mathrm{CH}_{3} \mathrm{CN} / \mathrm{H}_{2} \mathrm{O} / 0.1 \%$ TFA, 4 min gradient $)>95 \%$ pure by UV $(214 \mathrm{~nm})$ and $100 \%$ pure by ELSD. ${ }^{1} \mathrm{H}$ NMR (400, DMSO$\left.d_{6}\right) \delta 12.50(\mathrm{~s}, 1 \mathrm{H}), 8.02(\mathrm{~d}, J=8.9 \mathrm{~Hz}, 1 \mathrm{H}), 8.02(\mathrm{~d}, J=8.9 \mathrm{~Hz}, 1 \mathrm{H}), 7.54(\mathrm{~m}, 2 \mathrm{H}), 7.49$ $(\mathrm{m}, 2 \mathrm{H}), 7.44(\mathrm{~d}, J=7.2 \mathrm{~Hz}, 1 \mathrm{H}), 7.42(\mathrm{~d}, J=7.2 \mathrm{~Hz}, 1 \mathrm{H}), 7.36(\mathrm{~m}, 1 \mathrm{H}), 7.30(\mathrm{~d}, J=7.6$ $\mathrm{Hz}, 1 \mathrm{H}), 7.28(\mathrm{~d}, J=7.6 \mathrm{~Hz}, 1 \mathrm{H}), 7.21(\mathrm{~m}, 1 \mathrm{H}), 7.05(\mathrm{~m}, 2 \mathrm{H}), 3.82(\mathrm{~s}, 3 \mathrm{H}) ; \mathrm{HRMS} \mathrm{m} / z$ $327.1483\left(\mathrm{C}_{22} \mathrm{H}_{18} \mathrm{~N}_{2} \mathrm{O}+\mathrm{H}^{+}\right.$requires 327.1492).

Entry 5: Analytical LCMS: single peak (2.273 min, $\mathrm{CH}_{3} \mathrm{CN} / \mathrm{H}_{2} \mathrm{O} / 0.1 \%$ TFA, 4 min gradient $)>95 \%$ pure by UV (214 nm) and $100 \%$ pure by ELSD. ${ }^{1} \mathrm{H}$ NMR (400, DMSO$\left.d_{6}\right) \delta 12.42(\mathrm{~s}, 1 \mathrm{H}), 11.99(\mathrm{~s}, 1 \mathrm{H}), 8.65(\mathrm{~d}, J=5.6 \mathrm{~Hz}, 2 \mathrm{H}), 7.93(\mathrm{~d}, J=7.6 \mathrm{~Hz}, 1 \mathrm{H}), 7.83$ $(\mathrm{d}, J=7.6 \mathrm{~Hz}, 1 \mathrm{H}), 7.79(\mathrm{~m}, 2 \mathrm{H}), 7.64(\mathrm{t}, J=7.6 \mathrm{~Hz}, 1 \mathrm{H}), 7.59(\mathrm{~d}, J=7.6 \mathrm{~Hz}, 2 \mathrm{H}), 7.52$ $(\mathrm{d}, J=8.0 \mathrm{~Hz}, 1 \mathrm{H}), 7.47(\mathrm{~m}, 2 \mathrm{H}), 7.41(\mathrm{t}, J=7.2 \mathrm{~Hz}, 2 \mathrm{H}), 7.43-7.23(\mathrm{~m}, 5 \mathrm{H}), 7.16(\mathrm{t}, J$ $=7.6 \mathrm{~Hz}, 1 \mathrm{H}) ; \mathrm{HRMS} m / z 413.1771\left(\mathrm{C}_{28} \mathrm{H}_{20} \mathrm{~N}_{4}+\mathrm{H}^{+}\right.$requires 413.1761$)$.

\section{Representative analytical data for entries in Table 3:}

Entry 1: Analytical LCMS: single peak (2.361 min, $\mathrm{CH}_{3} \mathrm{CN} / \mathrm{H}_{2} \mathrm{O} / 0.1 \%$ TFA, 4 min gradient $)>95 \%$ pure by UV $(214 \mathrm{~nm})$ and $100 \%$ pure by ELSD. ${ }^{1} \mathrm{H}$ NMR (400, DMSO$\left.d_{6}\right) \delta 12.87(\mathrm{~s}, 1 \mathrm{H}), 8.11(\mathrm{~d}, J=8.4 \mathrm{~Hz}, 1 \mathrm{H}), 8.10(\mathrm{~d}, J=7.2 \mathrm{~Hz}, 1 \mathrm{H}), 7.84(\mathrm{~s}, 1 \mathrm{H}), 7.73$ (s, 1H), $7.49(\mathrm{~d}, J=7.2 \mathrm{~Hz}, 1 \mathrm{H}), 7.47(\mathrm{~d}, J=6.4 \mathrm{~Hz}, 1 \mathrm{H}), 7.41(\mathrm{~m}, 1 \mathrm{H}), 6.96(\mathrm{~d}, J=3.2$ 
$\mathrm{Hz}, 1 \mathrm{H}), 6.75(\mathrm{~d}, J=3.2 \mathrm{~Hz}, 1 \mathrm{H}), 6.67(\mathrm{~m}, 1 \mathrm{H}), 6.58(\mathrm{~m}, 1 \mathrm{H}) ; \mathrm{HRMS} m / z 277.0974$ $\left(\mathrm{C}_{17} \mathrm{H}_{12} \mathrm{~N}_{2} \mathrm{O}_{2}+\mathrm{H}^{+}\right.$requires 277.0972).

Entry 3: Analytical LCMS: single peak (2.537 min, $\mathrm{CH}_{3} \mathrm{CN} / \mathrm{H}_{2} \mathrm{O} / 0.1 \%$ TFA, 4 min gradient) $>95 \%$ pure by UV (214 $\mathrm{nm}$ ) and $100 \%$ pure by ELSD. ${ }^{1} \mathrm{H}$ NMR (400, DMSO$\left.d_{6}\right) \delta 12.50(\mathrm{~s}, 1 \mathrm{H}), 8.05(\mathrm{~d}, J=7.2 \mathrm{~Hz}, 2 \mathrm{H}), 7.45-7.38(\mathrm{~m}, 6 \mathrm{H}), 7.35(\mathrm{t}, J=7.2 \mathrm{~Hz}, 1 \mathrm{H})$, $7.01(\mathrm{~m}, 2 \mathrm{H}), 6.89(\mathrm{~m}, 2 \mathrm{H}), 3.79(\mathrm{~s}, 3 \mathrm{H}), 3.76(\mathrm{~s}, 3 \mathrm{H}) ; \mathrm{HRMS} m / 2357.1610\left(\mathrm{C}_{23} \mathrm{H}_{20} \mathrm{~N}_{2} \mathrm{O}_{2}\right.$ $+\mathrm{H}^{+}$requires 357.1598$)$.

Entry 5: Analytical LCMS: single peak (2.738 min, $\mathrm{CH}_{3} \mathrm{CN} / \mathrm{H}_{2} \mathrm{O} / 0.1 \%$ TFA, 4 min gradient) $>95 \%$ pure by UV (214 $\mathrm{nm}$ ) and $100 \%$ pure by ELSD. ${ }^{1} \mathrm{H}$ NMR (400, DMSO$\left.d_{6}\right) \delta 12.56(\mathrm{~s}, 1 \mathrm{H}), 8.06(\mathrm{~d}, J=7.2 \mathrm{~Hz}, 2 \mathrm{H}), 7.48-7.43(\mathrm{~m}, 4 \mathrm{H}), 7.39-7.36(\mathrm{~m}, 3 \mathrm{H}), 7.24$ (d, $J=8.0 \mathrm{~Hz}, 2 \mathrm{H}), 7.11(\mathrm{~d}, J=8.0 \mathrm{~Hz}, 2 \mathrm{H}), 2.35$ (s, 3H), 2.29 (s, 3H); HRMS m/z $325.1714\left(\mathrm{C}_{23} \mathrm{H}_{20} \mathrm{~N}_{2}+\mathrm{H}^{+}\right.$requires 325.1699).

\section{Lepidiline B:}

Acetaldehyde ( $34 \mu \mathrm{L}, 0.6 \mathrm{mmol}), 2,3$-butanedione $(53 \mu \mathrm{L}, 0.6 \mathrm{mmol})$, and ammonium acetate $(462 \mathrm{mg}, 6.0 \mathrm{mmol}$ ) were combined and dissolved in $3.0 \mathrm{~mL}$ of HOAc in a $5 \mathrm{~mL}$ Smithsynthesizer ${ }^{\mathrm{TM}}$ reaction vial (Part \#352016) containing a magnetic stir bar. The reaction vessel was heated in the Smithsynthesizer ${ }^{\mathrm{TM}}$ reactor cavity for $5 \mathrm{~min}$ at $180^{\circ} \mathrm{C}$, after which the vessel was rapidly cooled to $40{ }^{\circ} \mathrm{C}$ by the unit. The reaction mixture was added dropwise to a $0{ }^{\circ} \mathrm{C}$ concentrated $\mathrm{NH}_{4} \mathrm{OH}$ solution and was diluted with $20 \mathrm{~mL} \mathrm{H}_{2} \mathrm{O}$ before being extracted with EtOAc $(3 \times 20 \mathrm{~mL})$. The combined organic extracts were dried $\left(\mathrm{Na}_{2} \mathrm{SO}_{4}\right)$ and evaporated to afford 2,4,5-trimethylimidazole as a crystalline solid (50 mg, 76\%). Analytical LCMS: single peak (0.3 min, $\mathrm{CH}_{3} \mathrm{CN} / \mathrm{H}_{2} \mathrm{O} / 0.1 \%$ TFA, $4 \mathrm{~min}$ gradient $)>95 \%$ pure by UV $(214 \mathrm{~nm})$ and $100 \%$ pure by ELSD. ${ }^{1} \mathrm{H}$ NMR $\left(400, \mathrm{CDCl}_{3}\right)$ $\delta 2.31(\mathrm{~s}, 3 \mathrm{H}), 2.11(\mathrm{~s}, 6 \mathrm{H})$.

Benzyl chloride (127 mg, $1.0 \mathrm{mmol}$ ), 2,4,5-trimethylimidazole ( $22 \mathrm{mg}, 0.2 \mathrm{mmol}$ ), and $\mathrm{Et}_{3} \mathrm{~N}(141 \mu \mathrm{L}, 1.0 \mathrm{mmol})$ were combined and dissolved in $1.0 \mathrm{~mL}$ of $\mathrm{CH}_{3} \mathrm{CN}$ in a $2 \mathrm{~mL}$ Smithsynthesizer ${ }^{\mathrm{TM}}$ reaction vial (Part \#352016) containing a magnetic stir bar. The reaction vessel was heated in the Smithsynthesizer ${ }^{\mathrm{TM}}$ reactor cavity for $5 \mathrm{~min}$ at $180^{\circ} \mathrm{C}$, then rapidly cooled to $40{ }^{\circ} \mathrm{C}$ by the unit before being purified directly by HPLC to afford lepidiline B $(25 \mathrm{mg}, 43 \%)$ identical in all respect to properties reported for the natural product. ${ }^{1}$ Analytical LCMS: single peak (2.441 min, $\mathrm{CH}_{3} \mathrm{CN} / \mathrm{H}_{2} \mathrm{O} / 0.1 \%$ TFA, 4 min gradient) $>95 \%$ pure by UV $(214 \mathrm{~nm})$ and $100 \%$ pure by ELSD. ${ }^{1} \mathrm{H}$ NMR (DMSO- $\left.d_{6}\right) \delta$ 7.43-7.34 (m, 6H), $7.16(\mathrm{~d}, J=7.2 \mathrm{~Hz}, 4 \mathrm{H}), 5.45(\mathrm{~s}, 4 \mathrm{H}), 2.64(\mathrm{~s}, 3 \mathrm{H}), 2.13(\mathrm{~s}, 6 \mathrm{H}) ;{ }^{13} \mathrm{C}$ NMR (DMSO- $d_{6}$ ) $\delta 143.7,134.4,129.0$ (2C), 128.1, 126.5 (2C), 125.9 (2C), 47.9, 10.2, $8.3(2 \mathrm{C})$; HRMS $m / z 291.1885\left(\mathrm{C}_{20} \mathrm{H}_{23} \mathrm{~N}_{2} \mathrm{Cl}-\mathrm{Cl}^{-}\right.$requires 291.1856).

\section{Trifenagrel:}

Benzil (42 mg, $0.2 \mathrm{mmol}$ ), 2-[2-(dimethylamino)ethoxy]benzaldehyde ${ }^{2}$ (39 mg, 0.2 $\mathrm{mmol}$ ), and ammonium acetate (154 $\mathrm{mg}, 2.0 \mathrm{mmol}$ ) were combined and dissolved in 1.0 $\mathrm{mL}$ of HOAc in a $2 \mathrm{~mL}$ Smithsynthesizer ${ }^{\mathrm{TM}}$ reaction vial (Part \#352016) containing a magnetic stir bar. The reaction vessel was heated in the Smithsynthesizer ${ }^{\mathrm{TM}}$ reactor cavity for $5 \mathrm{~min}$ at $180^{\circ} \mathrm{C}$, after which the vessel was rapidly cooled to $40{ }^{\circ} \mathrm{C}$ by the unit. The reaction mixture was added dropwise to a $0{ }^{\circ} \mathrm{C}$ concentrated $\mathrm{NH}_{4} \mathrm{OH}$ solution and immediately formed a white precipitate which was collected by filtration and washed with $\mathrm{H}_{2} \mathrm{O}$. The solid was dried in a vacuum oven for $18 \mathrm{~h}$ at $50{ }^{\circ} \mathrm{C}$ to afford analytically 
pure trifenagrel (76 mg, 99\%). Analytical LCMS: single peak (2.109 min, $\mathrm{CH}_{3} \mathrm{CN} / \mathrm{H}_{2} \mathrm{O} / 0.1 \%$ TFA, 4 min gradient $)>95 \%$ pure by $\mathrm{UV}(214 \mathrm{~nm})$ and $100 \%$ pure by ELSD. ${ }^{1} \mathrm{H}$ NMR $\left(300, \mathrm{CDCl}_{3}\right) \delta 12.21(\mathrm{~s}, 1 \mathrm{H}), 8.47(\mathrm{dd}, J=1.8,7.8 \mathrm{~Hz}, 1 \mathrm{H}), 7.65(\mathrm{~d}, J$ $=7.2 \mathrm{~Hz}, 2 \mathrm{H}), 7.48(\mathrm{~d}, J=6.3 \mathrm{~Hz}, 2 \mathrm{H}), 7.40-7.26(\mathrm{~m}, 8 \mathrm{H}), 7.11(\mathrm{t}, J=7.5 \mathrm{~Hz}, 1 \mathrm{H}), 7.00$ $(\mathrm{d}, J=8.4 \mathrm{~Hz}, 1 \mathrm{H}), 4.23(\mathrm{dd}, J=5.1,6.0 \mathrm{~Hz}, 2 \mathrm{H}), 2.67(\mathrm{dd}, J=5.1,5.4 \mathrm{~Hz}, 2 \mathrm{H}), 1.97(\mathrm{~s}$, $6 \mathrm{H})$; HRMS $m / z 384.2070\left(\mathrm{C}_{25} \mathrm{H}_{25} \mathrm{~N}_{3} \mathrm{O}+\mathrm{H}^{+}\right.$requires 384.2070).

(1) Cui, B.; Zheng, B. L.; He, K.; Zheng, Q. Y. J. Nat. Prod. 2003, 66, 1101.

(2) Phillips, A. P.; White, H. L.; Rosen, S. Eur. Patent 58890(A1), 1982. 